Jean-Claude Hocquet, Jean-Luc Sarrazin (dir.), Le Sel de la Baie : histoire, archéologie, ethnologie des sels atlantiques

Presses universitaires de Rennes, Rennes, coll. « Histoire », 2006, 411 p.

\title{
Alain Gallicé
}

\section{OpenEdition}

Journals

Édition électronique

URL : https://journals.openedition.org/abpo/92

DOI : $10.4000 / a b p o .92$

ISBN : 978-2-7535-1506-2

ISSN : 2108-6443

Éditeur

Presses universitaires de Rennes

Édition imprimée

Date de publication : 30 juin 2007

Pagination : 196-202

ISBN : 978-2-7535-0510-0

ISSN : 0399-0826

Référence électronique

Alain Gallicé, « Jean-Claude Hocquet, Jean-Luc Sarrazin (dir.), Le Sel de la Baie : histoire, archéologie, ethnologie des sels atlantiques ", Annales de Bretagne et des Pays de l'Ouest [En ligne], 114-2 | 2007, mis en ligne le 31 décembre 2009, consulté le 22 juillet 2022. URL : http://journals.openedition.org/abpo/ 92 ; DOI : https://doi.org/10.4000/abpo.92

Ce document a été généré automatiquement le 22 juillet 2022.

Tous droits réservés 


\section{Jean-Claude Hocquet, Jean-Luc Sarrazin (dir.), Le Sel de la Baie : histoire, archéologie, ethnologie des sels atlantiques}

Presses universitaires de Rennes, Rennes, coll. « Histoire », 2006, 411 p.

\section{Alain Gallicé}

\section{RÉFÉRENCE}

Presses universitaires de Rennes, Rennes, coll. « Histoire », 2006, 411 p.

\section{NOTE DE L'ÉDITEUR}

Cet ouvrage sur le site des Presses universitaires de Rennes : notice, documents et commande en ligne : http://www.pur-editions.fr/detail.php?idOuv=1209

1 Ce livre est l'édition des actes du colloque Le Sel de la Baie et ses concurrents,tenu à Nantes et à Batz-sur-Mer les 16, 17 et 18 septembre 2004 et organisé par le CRHIA (Centre de recherches en histoire internationale et atlantique-université de Nantes) en partenariat avec le CERSATES (UMR-CNRS 8529-université Charles de Gaulle, Lille III) et le musée des Marais salants (CAP Atlantique), sous le patronage de la Commission internationale d'histoire du sel.

2 Par sel de la Baie, il faut entendre Baiensolt, Bayen salz, Bay salt, dénomination empruntée aux Hanséates et aux Anglo-Saxons et désignant l'ensemble des sels marins atlantiques. Grâce aux vingt-cinq contributions, d'horizons divers, son histoire, déjà largement défrichée, s'approfondit et se renouvelle, le dialogue instauré entre historiens, géographes et archéologues se révélant extrêmement fructueux. 
3 En introduction, Jean-Claude Hocquet met en perspective l'histoire et rappelle que si ses permanences sont considérables, ses mutations n'en sont pas moins fortes. Les révolutions industrielles, techniques et des transports favorisent d'abord les salines solaires atlantiques dont le sel gagne l'Europe du Nord et anime un vaste trafic maritime, avant que cette zone ne devienne productrice de sel à l'époque industrielle, ce qui provoque le déclin des marais salants atlantiques dont, de nos jours, certains connaissent un mouvement de reprise.

4 L'ouvrage s'ordonne en cinq parties qui ont pour thème: les sels marins avant les marais salants; la production des sels atlantiques : les sociétés saunières et les modes de faire-valoir salicoles; les transformations et commercialisations des sels atlantiques ; les concurrences et circulations des sels; les perspectives comparatives.

5 La première partie s'intéresse aux sels marins avant les marais salants. L'archéologie est en mesure de préciser la date et les conditions des premières productions. Serge Cassen, dans un article cosigné avec Jean-Arnaud de Labriffe et Loïc Ménanteau, montre, par une analyse comparative, que, dès le $\mathrm{V}^{\mathrm{e}}$ millénaire av. J.-C., existent des systèmes de chauffage des saumures. Cette activité n'a pu que bouleverser le régime socio-politique des sociétés indigènes et pourrait être un des fondements de l'extraordinaire développement d'architectures funéraires monumentales du sudArmorique.

6 Une fouille menée à Vigo renseigne sur l'origine des salines solaires atlantiques. Juan C. Castro Carrera décrit un type de saline romaine d'évaporation qui fait figure de chaînon manquant dans l'évolution conduisant aux marais salants. Les évaporateurscristallisoirs présentent une structure empierrée dont les bassins, tapissés d'une fine couche d'argile imperméable, sont séparés par d'étroits murets très semblables à ceux de certaines salines continentales actuelles.

7 À l'échelon d'une région, des synthèses s'élaborent. Par l'analyse des produits dont le sel est un composant (métallurgie, ateliers de salaisons, fours d'amphores à salaisons), Nuria Morère retrace l'histoire du sel atlantique hispanique pendant l'Antiquité. Au premier millénaire, Cadix domine la production, puis au Bas-Empire est relayé par Huelva, la Galice et la côte du Portugal. Durant l'Antiquité tardive, les petites installations se multiplient témoignant d'une économie orientée vers la consommation locale.

8 Comprendre la localisation des sites et déterminer les techniques de fabrication du sel s'avèrent souvent délicats. Carlos Alonso et Loïc Ménanteau soulignent les apports de la restitution des paléopaysages et des paléoclimats pour déterminer le contexte des zones étudiées, alors que la recherche archéométrique de restes d'aliments salés ou d'échantillons de sel ancien peut aider à préciser la technologie employée - un indicateur fiable pourrait être le brome, présent dans l'eau de mer mais absent chez les êtres vivants et dans le sel solaire.

9 Ces contributions attestent qu'une archéologie de la production salicole est désormais expérimentée.

10 Les sources écrites gardent leur importance. Cristina Carusi esquisse une histoire des politiques d'exploitation du sel dans le monde grec. Les conditions climatiques et environnementales n'ont pas incité les poleis à s'occuper d'exploitation du sel qui n'a pas fait pas l'objet de dispositions législatives spécifiques, sauf exception. En Égypte, sous les Lagides, le sel, comme les principaux produits naturels, appartient au roi qui en 
contrôle le commerce. Le sel peut être à la base d'un impôt, attesté pour le royaume des Séleucides.

11 La deuxième partie de l'ouvrage traite des sociétés saunières et les modes de fairevaloir salicoles. Elle illustre un des renouvellements majeurs de la recherche. Longtemps, les études historiques ont favorisé les commerces terrestres et maritimes, les espaces et les réseaux de la diffusion et la fiscalité étatique, faisant de l'État, dans sa logique fiscale, et de la demande extérieure, dans ses composantes structurelles, les régulateurs de la production. Désormais, l'attention se porte sur les techniques de production, les modes de faire-valoir, la hiérarchie au sein de la paysannerie saunière, sur l'intérêt des acteurs sociaux (propriétaires, fermiers, sauniers, manouvriers) pour la gestion des marais salants. Au cœur de la recherche se placent les marais salants en eux-mêmes et leurs sociétés en prenant en compte les aspects techniques, sociaux, économiques, les contraintes du milieu et les options choisies par les détenteurs du sol, bref, les logiques internes des sociétés salicoles.

Julien Briand, grâce à un document exceptionnel - un cahier de comptes couvrant la période 1463-1512 et concernant les possessions acquises par les Blanchet, une famille d'officiers de justice nantais -, éclaire les structures de production, les sociétés saunières et la rentabilité économique d'une exploitation de salines dans les pays bretons de la baie de Bourgneuf à la fin du Moyen Âge. Si le schéma de base du mode de production parait simple, un tiers de la récolte pour le saunier, deux tiers pour le maître qui prend en charge les frais d'entretien et les taxes seigneuriales, il donne lieu à de multiples possibilités toujours défavorables au saunier, dont l'endettement conduit à son assujettissement.

13 Les sources fiscales demeurent une mine d'informations. À partir de deux rôles d'imposition (1786 et 1787-1789), Philippe Bossis étudie la société saulnière en Olonnais. Sur les 8500 habitants du bassin-versant des marais d'olonne, 2200 travaillent à la production du sel. Pour les impositions ordinaires, la cote moyenne des saulniers est de $61.14 \mathrm{~s}$. (7,5 fois celle du niveau de base, celui du journalier), signe d'une modeste aisance. Cependant, la société saunière est inégalitaire : seuls 17 \% d'entre eux peuvent être électeurs et un sur cinq prétendre être élu maire ou officier municipal.

Les mutations technologiques sont un moment clé pour apprécier le rôle des différents acteurs. Claude Bouhier revient sur le transfert de technologie saunière de Guérande à Noirmoutier, qui voit, au tout début $\mathrm{du}$ XVIII ${ }^{\mathrm{e}}$ siècle, les propriétaires noirmoutrins abandonner le système des aires saunantes pour celui les œillets. Les considérations financières sont déterminantes, il s'agit d'obtenir un meilleur rendement et une seule qualité de sel dans le cadre d'un trafic local, simplifié et lié à un client privilégié : la Ferme générale. La présence de représentants de la grande noblesse de cour, liés à la Ferme, possessionnés dans les marais de la Baie favorise le mouvement.

15 Le paysage se transforme et justifie une approche paysagière. Sarah Réault-Mille met en rapport les paysages et les techniques salicoles dans un essai de lecture géo-ethnohistorique des marais salants du littoral de la Charente-Maritime. Les traces paysagères, confrontées aux sources archéologiques, historiographiques, archivistiques et toponymiques, permettent d'établir une morpho-chronologie paysagère comportant cinq types correspondant à cinq périodes différentes. Au fil du temps, la taille des salines croît et leur morphologie se géométrise et se complexifie signes d'un fort évolutionnisme technique. 

d'Alveiro (Portugal) à la fin du XvII siècle et au cours du xvIII siècle. Inês Amorin en fait le point. Autour de 1700, la petite propriété morcelée domine. La noblesse n'en détient qu'une faible proportion de salines (13\%), les religieux sont présents (34\%), mais l'essentiel revient à l'élite locale des notables, citoyens et administratifs (47\%). L'exploitation, assurée par des métayers disposant d'un contrat annuel, permet en abaissant les coûts de reconstruction des salines, l'élan de la production et la réussite commerciale.

Autre renouvellement de la recherche, celui qui met l'accent sur l'insertion de la production salicole dans les écosystèmes. Jean-Pierre Corlay, à partir de l'exemple des marais salants de Guérande, replace les enjeux de la saliculture actuelle dans le cadre du développement durable. En déclin depuis le milieu du XIXe siècle, le marais est menacé de disparaître à la fin des années 1960. Il est sauvé par une mobilisation locale et, depuis 1996, le classement du site garantit la protection du territoire. La devise, inventée en 1988-1989 « un site : le marais; des hommes : les paludiers; un produit : le sel » traduit une démarche visant à construire un espace-projet, ce qui évoque le trépied conceptuel du développement durable, donnant à la presqu'île guérandaise valeur de défense et d'illustration de celui-ci.

18 La troisième partie de l'ouvrage a pour objet les transformations et commercialisations des sels atlantiques. Les thèmes traditionnels (routes et marchés, enjeux de la pénétration commerciale, mutations du commerce, aléas de la conjoncture, fiscalité) bénéficient d'un élargissement des sources prises en compte.

Une approche numismatique s'avère riche de perspectives. Olivier Bruand le montre pour le haut Moyen Âge. L'étude des monnaies diffusées à partir des zones salicoles et celles mises en circulation dans l'intérieur des terres ou sur des côtes éloignées des marais salants, et retrouvées en ces lieux, donnent des résultats qui confortent ceux obtenus à partir des sources écrites (franchises de tonlieux ou récits hagiographiques). Ils attestent d'un trafic saunier actif dès les $\mathrm{VI}^{\mathrm{e}}$ et $\mathrm{VII}^{\mathrm{e}}$ siècles selon des itinéraires que l'on suit jusqu'au début du $\mathrm{x}^{\mathrm{e}}$ siècle. Les sauniers de la Baie, dont la montée en puissance s'amorce dès l'époque mérovingienne, et, dans une moindre mesure, ceux d'Aunis et de Saintonge, à l'époque carolingienne, chargent les navires livrant du sel vers les côtes normandes, picardes, anglaises, les bouches de la Seine et de la Somme, voire vers la Flandre et la Frise. Par terre, le sel gagne les pays de la Loire, de la Seine, le Limousin et la vallée de la Garonne.

Surtout, à partir du commerce maritime, la recherche s'ouvre à l'étude des arrière-pays portuaires, des marchés de base, des marchands de sel, des compagnies de marchands, à l'impact de la saliculture dans le développement régional.

21 À l'échelle d'un territoire, la place relative du sel et du vin, deux grands produits du commerce international médiéval, est étudiée par Mathias Tranchant dans le cadre de l'économie rochelaise. Sur la foi des cartulaires ecclésiastiques du Centre-Ouest, la saliculture connaît son véritable décollage au cours des $\mathrm{xe}$ et xie siècles, pour disparaître des sources dès le début du xile siècle. Certes, elle n'a pas disparu, mais elle végète. Le sel et le vin ne peuvent être produits à grande échelle dans un même secteur jusqu'au milieu du xve siècle. Leurs besoins en capitaux et en main-d'œuvre obligent les acteurs économiques locaux à privilégier l'une des deux productions. La naissance de La Rochelle, fondée notamment sur le commerce du vin, plus rémunérateur, vide, en 
partie, le secteur salicole dès le xIIIe siècle; seules, les aires saunières saintongeaises gardent une production.

Au Moyen Âge, l'enjeu des privilèges liés au sel est souligné par Michel Bochaca. À Libourne, les premières mentions écrites de droits levés sur le sel et de privilèges sur son trafic datent des années 1340-1350. Alors, se met en place un système d'étape faisant de Libourne le point de passage et de transit obligé du commerce par la Dordogne. Entre 1470 et 1525, face aux prétentions des villes et des marchands de la basse vallée de la Dordogne, les Libournais n'ont de cesse de défendre leurs privilèges et leur grenier à sel. En cela, ils visent la conservation des revenus municipaux mais, plus largement, de tout un système économique dont le grenier à sel, et le trafic qu'il draine, constitue la pierre angulaire.

L'apport des actes notariés se révèle particulièrement fécond. Les archives notariales nantaises aident à préciser la destinée largement méconnue des salines de la baie de Bourgneuf au XVII ${ }^{e}$ siècle. La source est incomplète et déforme la réalité en faisant la part belle aux actes passés par des Nantais et des habitants de la baie de Bourgneuf et le trafic du sel destiné à Nantes et aux provinces de l'intérieur, mais, pour Bernard Michon, elle désigne le principal débouché du sel de la Baie: si le commerce avec l'étranger paraît dominer jusqu'au milieu du xvII ${ }^{\mathrm{e}}$ siècle, le fait que la place de sel de la Baie, sur le marché nantais, devienne prépondérante à partir de ce moment-là est le signe d'une évolution.

Une autre enquête menée dans les actes notariés nantais, concernant les deux premières décennies du règne de Louis XIV, révèle un important trafic de sel des fermiers espagnols de la fiscalité salicole pour alimenter leurs greniers à sel des royaumes des Asturies et de Galice alors que la proximité géographique des salines portugaises et l'importance des marchands portugais dans le commerce de la côte cantabrique favorisent les sels portugais. D'autre part, à Nantes, depuis 1580, l'importance du sel dans le trafic commercial n'a pas cessé de se réduire jusqu'à un seuil sans doute atteint dans les années 1670 , alors que, si à partir du XIV siècle l'Espagne constitue le premier horizon international du port, le développement des liens avec l'Europe du Nord, sous l'impulsion des marchands hollandais, renverse cette hiérarchie dans le premier tiers du XviI e siècle. Pour Guy Saupin, ce commerce du sel a un caractère conjoncturel, il renvoie aux conséquences de la guerre d'indépendance menée par le Portugal contre l'Espagne (1640-1668). Après 1668, dans une sorte de sanction économique, la Couronne espagnole cherche une revanche. La guerre de Hollande anéantit ce recours aux marais français et rétablit des anciens courants commerciaux.

Grâce à des sources diversifiées, Gildas Buron réagit à l'opinion couramment exprimée selon laquelle la production salicole guérandaise n'a connu, aux Temps modernes, de débouchés que régionaux vers la Bretagne intérieure. Au XVII siècle, elle est l'objet d'un commerce international, largement dominé par les Anglais, Écossais et Irlandais, ce qui n'exclut pas la présence de Flamands, Hollandais et de représentants des Nations du Nord, alors qu'Espagnols, Portugais et Basques figurent parmi les clients réguliers. Avec l'Europe du Nord, les sels de Guérande n'ont de véritable succès qu'au XviII ${ }^{\mathrm{e}}$ siècle, la décennie 1741-1750 connaissant un pic d'activité (1 015 passages de navires chargés de sel de Guérande franchissent le Sund). Cette poussée s'affirme après le déclin du port de Brouage et avec l'affirmation de la marine hollandaise qui s'impose lors de la guerre de Succession d'Autriche, pour dominer ensuite, jusqu'aux années 1760, le transport des 
sels bretons vers la Baltique, avant d'être supplantée par les Scandinaves, Suédois puis Norvégiens.

La quatrième partie est consacrée aux concurrences et circulations du sel. L'attention est portée sur les dynamiques microrégionales et les espaces élémentaires en relation avec les grands réseaux. Il en résulte une complexification des schémas jusqu'alors proposés.

Ainsi le royaume de Grenade dispose, au XII eau $\mathrm{XV}^{\mathrm{e}}$ siècle de salines maritimes et continentales, mais, selon Antonio Malpica Cuello, fait appel à des importations en provenance des salines atlantiques pour répondre aux besoins des pêcheries et des salaisons.

Au Moyen Âge, de la Frise à la Prusse, le sel de la Baie est en compétition avec le sel de Lunebourg. Dès 1407, face à la concurrence, tout commerce de transit de sel étranger par le duché de Lunebourg est interdit, et, en 1495, Lunebourg, renouvelant les plaintes exprimées en 1405 relatives à l'usage frauduleux de ses tonneaux, prend des dispositions réglementaires. Harald Witthöt montre que le marché du sel de Lunebourg, n'est pas homogène, mais constitué d'un faisceau de branches ayant chacune son développement et sa structure, le prix n'étant pas le seul élément qui fonde la concurrence, entrent également en jeu la disponibilité, la qualité du produit et la spécificité de la demande.

$\mathrm{Au}$ XVIII ${ }^{\mathrm{e}}$ siècle, sur ces marchés du Nord, les sels ibériques français et britanniques sont en concurrence. Pierrick Pourchasse établit que jusqu'au début des années 1770, les importations de sel en Baltique sont dominées par les productions française et portugaise. Les exportations françaises atteignent leur maximum au cours de la décennie 1740-1749, puis stagnent pendant une vingtaine d'années, avant de chuter à partir de 1770. À la fin du siècle, le Portugal et le Royaume-Uni triomphent, sauf en Scandinavie. Ce déclin des sels français s'explique par une série de mauvaises récoltes au cours de la période 1763-1774. Le dynamisme commercial britannique est un autre facteur : depuis le milieu du XVIII ${ }^{\mathrm{e}}$ siècle, le Royaume-Uni exporte un sel, produit sur des bases industrielles dans les mines de Cheshire, meilleur marché que le sel français, et qui représente, pour les négociants britanniques, un fret de départ vers la Baltique. Enfin, au cours de la guerre d'Amérique, le Royaume-Uni en vient à contrôler la route de la Baltique, au détriment des Hollandais.

La complexité des facteurs en jeu dans la concurrence entre les sels est encore illustrée par Carol D. Litchfield à propos de l'Amérique du Nord au XVIII ${ }^{\mathrm{e}}$ et XIX ${ }^{\mathrm{e}}$ siècle. Au temps des colonies anglaises, le sel est interdit de production, et doit être acheté à la mèrepatrie. Lors de la guerre d'Indépendance, les colonies installent de petites salines et ont recours au sel des Caraïbes. Après la période révolutionnaire, les États-Unis importent d'Espagne, de France et du Portugal. Ce sel, utilisé comme fret de départ par des navires qui gagnent les États-Unis, est, dans ce pays, préféré pour la conservation de la viande et du poisson et pour le saumurage. Toutefois, les États-Unis se dotent d'une production nationale d'un grand nombre de types de sels qui entrent en compétition avec celui importé. Des taxes douanières, à caractère protectionniste, sont établies, puis, supprimées en 1913.

31 La cinquième partie ouvre des perspectives comparatives. Yannis C. Saïtas et Cornelia I. Zarkia décrivent la récolte du sel dans la péninsule de Magne (Péloponnèse). Leur étude, ethnologique, a été enrichie, par Jean-Claude Hocquet, d'un contenu historique. 
En effet, ces salines constituent un apport capital sur les techniques de production du sel avant les marais salants dans une zone, la Méditerranée, où les origines de la culture $\mathrm{du}$ sel restent une question ouverte. Ce sont des salines archaïques, à la fois rudimentaires (rochers creusés) et complexes (fonctions différenciées des bassins), qui ont survécu, dans un site rocheux inhospitalier et protégé, depuis les temps préhistoriques.

Alain Venturi livre une étude comparative du sel des pays d'Aigues-Mortes (royaume de France) et de Camargue proprement dite (comté de Provence, Empire) du $\mathrm{XI}^{\mathrm{e}} \mathrm{au}$ $\mathrm{xv}^{\mathrm{e}}$ siècle. Face aux salines du Languedoc, de l'étang de Berre ou de celles de Fos, celles de Camargue ont souffert d'une médiocrité initiale qu'expliquent des problèmes techniques liés à la maîtrise des eaux du Rhône et à un relatif isolement. Cependant, les salines du comté de Provence se développent, puis celles autour d'Aigues-Mortes (dites de Peccais) après la fondation de la ville en 1246. En 1301, le roi de France et Charles III d'Anjou établissent une societas pour le tirage, par le Rhône, du sel provenant des salines domaniales de Peccais et de celles de Provence. Au XIV siècle, les guerres portent un coup au commerce des sels de Provence, mais il faut attendre le début du $\mathrm{xvI}^{\mathrm{e}}$ siècle pour que les salines de Peccais fournissent les trois-quarts de l'ensemble du tirage de l'Empire et du Royaume.

Dans sa conclusion, Jean-Luc Sarrazin note que le colloque a dressé un bilan aussi exhaustif que possible des recherches actuelles et que ses apports sont essentiels concernant la commercialisation, les modes sociaux de production salicole, les types d'aménagement et de développement territorial. En annexe, Jean-Claude Hocquet revient sur le mesurage des sels sur les marais de l'Atlantique français.

Regrettons que, faute de temps, deux communications n'ont pu être traduites, que les cinq parties soient disproportionnées et, encore, que la perspective comparative n'ait pas été étendue au rivage atlantique du Maroc éclairé par des travaux récents ${ }^{1}$. Avec Jean-Luc Sarrazin, soulignons également l'absence de contributions relatives au Moyen Âge central; et plus encore, aux marchés de la terre salicole et de la rente durant la période $\mathrm{XIII}^{\mathrm{e}}-\mathrm{XIX}{ }^{\mathrm{e}}$ siècle, études qui permettraient de mieux cerner l'articulation entre production et commercialisation, de mesurer l'impact de la saliculture sur le développement régional et de mieux saisir l'importance des investissements régionaux et urbains dans la saliculture.

\section{NOTES}

1. Entre autres, HESNARD, Antoinette, «Le sel des plages (Coatta et Taldart, Maroc) ", Mélanges de l'École française de Rome, Moyen Âge, 1998, p. 167-192. 